\title{
Medication and tooth movement
}

\author{
Abstracted from \\ Bartzela T, Türp JC, Motschall E, Maltha JC. \\ Medication effects on the rate of orthodontic tooth movement: a systematic literature review. \\ Am J Orthod Dentofacial Orthop 2009; 135: 16-26 \\ Address for correspondence: Jaap C Maltha, Radboud University Nijmegen Medical Centre, \\ Department of Orthodontics and Oral Biology, 309 Tandheelkunde, Postbus 9101, NL 6500 HB Nijmegen, \\ The Netherlands. E-mail: j.maltha@dent.umcn.nl
}

\section{Question: Can an individual's medication affect the rate of orthodontic tooth movement?}

Data sources PubMed, Embase, Web of Science and Biosis were used to search for relevant material, along with the reference lists of retrieved articles.

Study selection Both animal and clinical studies were included if they met the following criteria: they had study and control groups of at least five subjects each; used drugs or supplements with known effects on bone physiology; recorded drug dose and administration; gave details of the application of the forces used for tooth movement; described the technique used to measure the rate of tooth movement; and presented a statistical analysis of the results of the study.

Data extraction and synthesis A formal data extraction process is not described.

Results Forty-nine studies were included in this review. Numerous problems were found in the information presented in the reviewed literature from the almost exclusively animal studies. Comparison of the data from these studies was difficult because of the variability in experimental design, animal models, administration regimens, application and duration of forces to the teeth involved.

Conclusions The authors identified a need for more well designed studies on the effects of various types of medication on orthodontic tooth movement.

\section{Commentary}

A systematic review on the effect of medication on rate of tooth movement is an indicator of a paradigm shift underway in orthodontics. As Thomas Kuhn so aptly stated, ${ }^{1}$ scientific and intellectual advances relate to the displacement of a paradigm that has become increasingly incapable of describing or explaining facts, and the move to a new paradigm that offers more satisfactory explanations. Here, a mechanistic paradigm is being increasingly displaced by a biological paradigm in orthodontic tooth movement (OTM). The realisation that analysing forces and vectors does not really explain the complex interaction of strain, gene expression, integrins and activation of signalling pathways defines this new paradigm and the search for new answers to old questions.

To be accepted as a paradigm, says Kuhn, a theory must be better than its competitors but it need not and in fact never does explain all the facts it faces. If one accepts this contention, then this systematic review of literature fulfils a useful role. The review is based on either animal or clinical investigations, with control and experi- mental groups and an adequate description of the nature of intervention, dosage, administration and the technique, and the force levels related to tooth movement.

The search strategy is comprehensive, using PubMed, Embase, Web of Science and Biosis, and the references of retrieved articles. There is also a mention of web-based information regarding medications and their effects on mediators (e.g. leukotrienes, thromboxanes, prostacyclins, and prostaglandins). A total of 49 studies have been included by the authors. It is at this point that one senses the difficulty faced by the reviewers with their data abstraction and synthesis. It is an ambitious review which has to deal with a wide disparity in study designs, regimens of administration, force levels and duration, leading to inconclusive outcomes and at best an inductive reasoning on the projected effects of medications.

The review is based on experimental data on the effects of pharmaceutical interventions and the use of dietary supplements on the rate of OTM. In the absence of human clinical trials, the review is based on well-controlled animal studies.

It is possible to categorise pharmaceutical interventions that would affect OTM into the four broad categories: nonsteroidal antiinflammatory drugs (NSAID), corticosteroids, other analgesics and bisphosphonates. There are a host of systemic factors and mediators, however, that could influence the rate of OTM. The authors have made a concerted effort to present a comprehensive picture.

The review addresses the systemic mediators before relating them to direct pharmaceutical interventions. Eicosanoids or prostanoids are pivotal for conversion to thromboxanes, prostacyclins and prostaglandins. It is the effect of synthetic analogues or inhibitors on these signalling molecules, which are critical to regulatory pathways and pathological responses, that directly or indirectly affects OTM. Both thromboxanes and prostacyclins are affected by NSAID and might inhibit the rate of OTM. Prostaglandins play an important role in inflammation and their effects have been studied before. Animal studies seem to suggest some acceleration of OTM but no convincing statistical or clinical correlation is possible. There is a positive trend to show that the exogenous prostaglandins such as misoprostol may correlate with an increased rate of OTM.

This does bring into focus the effects of NSAID on prostanoid synthesis. All NSAID have approximately the same effect and mechanism of action; they constitute the largest group of commonly used drugs with potential effects on orthodontic tooth movement. Cyclooxygenase-2 inhibitors have been withdrawn because of a possible correlation with increased risk of cardiac attacks and strokes, but salicylates, arylalkanoic acids such as diclofenac, arypropionic 
acids such as ibuprofen, and oxicams, do find long term usage in chronic conditions such as arthritis, cardiovascular conditions and cancer. The issue when relating these to OTM is that the period of administration is of a relatively short duration and presumably the majority of orthodontic patients would fall into the category of nonchronic usage. It is difficult for this review and any other published material to conclusively form the basis for recommendations relating to the rate of OTM.

Paracetamol seems to be the safest drug to prescribe when managing pain related to orthodontic treatment because well-designed animal studies suggest no effects on OTM. Corticosteroids may increase OTM.

Calcium and calcium regulators deserve and find a special mention in the review. Calcium homeostasis is affected by parathyroid hormone, thyroid hormone, oestrogens, vitamins and diet. A group of drugs that have a direct influence on calcium homeostasis are the bisphosphonates, increasingly often mentioned in the orthodontic literature. Calcium metabolism is intricately associated with general bone physiology and an interesting picture is emerging. Vitamin D3 stimulates tooth movement, whereas dietary calcium seems to inhibit it. The parathyroid hormone seems to stimulate the rate of OTM, and oestrogens probably reduce tooth movement. Bisphosphonates are increasingly being used in the management of osteoporosis, Paget's disease and bone metastasis. This is one group of drugs that would conclusively affect OTM. With an extremely long half-life of 10 years or more, they continue to affect bone metabolism for years after a patient has completed therapy. A possible application in managing relapse is fraught with risks of osteonecrosis. The only fortunate aspect is that the average orthodontic patient is not likely to be exposed to bisphosphonate therapy.
The picture that emerges from the review is hazy, with no clear boundaries being defined. Direct extrapolation of animal studies to clinical situations is not possible and the systematic review is based almost entirely on animal studies, albeit well-designed ones. Nevertheless, one can appreciate this review's contribution to the understanding of the basic biology of OTM, especially with the increasing number of patients at both ends of the age spectrum who seek orthodontic treatment, some of whom orthodontists will find require protracted medication for chronic ailments. Prescription drug use and over-the-counter medication is also expanding in the younger generation, in addition to the possibility of long-term drug (mis-) use . It is therefore important for the orthodontist to assume that at least some, if not a majority of patients, are taking some medication and that this could be a confounding factor in predicting treatment outcomes. The need for careful enquiries about prescription/ nonprescription medication and dietary supplements should be an integral part of the history taken when planning orthodontic treatment.

The authors should be complemented on undertaking a challenging review: this may not be a classic review in the evidencebased field but could be seminal in directing us to look beyond brackets and wires. After all, an emerging paradigm may not provide satisfactory answers to all questions but certainly provides clear perspectives and directions.

Anmol S Kalha

Institute of Dental Studies and Technologies, Modinagar, India

1. Kuhn T S, The Structure of Scientific Revolutions, 2nd. ed., Chicago: Univ. of Chicago Pr., 1970

Evidence-Based Dentistry (2009) 10, 50-51. doi:10.1038/sj.ebd.6400652 